\title{
Simple observations concerning black holes and probability *
}

\author{
Sándor Hegyi \\ KFKI Research Institute for Particle and Nuclear Physics \\ H-1525 Budapest, P.O. Box 49. Hungary
}

(Dated: October 30, 2018)

\begin{abstract}
What is common in a black hole and a bell-shaped curve? The question does not seem to make much sense but it is argued that black holes and the limit distributions of probability theory share several properties when their entropy and information content are compared. In particular the nohair theorem, the entropy maximization and holographic bound, and the quantization of entropy of black holes have their respective analogues for stable limit distributions. This observation suggests that the central limit theorem can play a fundamental role in black hole statistical mechanics and in a possibly emergent nature of gravity.
\end{abstract}

Keywords: Black holes, entropy, central limit theorem, stable distributions

\footnotetext{
* Essay awarded "Honorable Mention" in the Gravity Research Foundation 2009 Essay Competition
} 
Ever since the pioneering work of Bekenstein [1] and Hawking [2] black hole (BH) thermodynamics remained one of the most intensively studied subjects in gravity research $[3,4]$. This can be attributed to the fact that the thermal properties of BHs seem to have a fundamentally quantum gravitational origin: quantities such as the temperature and entropy of a black hole depend both on Newton's constant $G$ and Planck's constant $\hbar$. Interestingly, the several different approaches to the quantum theory of gravitation exhibit a profound universality [4]: at the microscopic level these considerations attribute the BH entropy to different microstates, nevertheless all of them correctly reproduce the Bekenstein-Hawking formula $S_{\mathrm{BH}}=A / 4$ relating the entropy $S_{\mathrm{BH}}$ of a black hole to the surface area $A$ of its event horizon measured in Planck units.

A possible cause of this surprising and not fully explained success may lie in the central limit theorem (CLT) of probability theory. The CLT provides the core of the mathematical foundations of statistical mechanics [5] and hence its utility in a deeper understanding of BH entropy would not be totally unexpected.

The essence of CLT is not that foreign to the realm of black holes. BHs are formed by the aggregation of masses until the limit of a gravitational collapse is reached. The CLT deals with aggregation of independent and identically distributed random variables and specifies the probability distribution of the properly normalized aggregate in the limit of an infinite number of its individual components [6]. The CLT tells that the common distribution of the aggregated degrees of freedom displays an overwhelming simplicity and universality. Approaching the limit, the details of the individual components' distribution progressively disappear and finally one arrives at a probability law characterized only by a few parameters. Moreover, the same limit law will emerge for infinitely many different choices of the aggregated components. If the first two moments of the individual distributions exist the limit law is the Gaussian specified fully by its mean and variance.

The fact that the limit laws obey a very simple shape irrespective of how complex is the distribution of the individual components is reminiscent of the no-hair theorem: externally a black hole is characterized only by its mass, electric charge and angular momentum. Any other information about the collapsing matter such as quantum numbers otherwise conserved in laboratory circumstances are not preserved by BHs. In case of the CLT the higher order fluctuations of the aggregated random variables are not preserved. These fluctuations, however large but finite, are progressively smoothed out as the number of 
individual components increases. As a result, for the emerging Gaussian only the first two cumulant moments exist (mean and variance) all the higher order cumulants characterizing higher fluctuations vanish.

The aggregation of random variables means convolution of their distributions. Convolution is an information burning operation, the entropy can not decrease under its repeated application. Therefore the limit distributions must have larger entropy than the individual components being aggregated. Indeed, the Gaussian law has maximum entropy among the probability densities of fixed variance. This was shown by Shannon in his classic paper setting the foundations of information theory [7].

From the no-hair theorem we know that there is an enormous information loss when black holes are formed. Accordingly BHs have much larger entropy than any known object of same mass. The entropy of black holes scales with the surface area, $S \sim A$, whereas non-BH objects satisfy the 't Hooft entropy bound $S<A^{3 / 4}[8]$. Recently it was pointed out that in the classical limit the maximum entropy of a quantized surface is proportional to its area for a wide class of surface quantization schemes [9]. Thus the BH horizon has maximum entropy among the spherical surfaces of fixed area. The similarity to limit laws is obvious with their variance or width playing the role of $\mathrm{BH}$ event horizon area. But it is early to conclude that the analogy has solid foundations. The possible existence of hyperentropic objects and so-called monster configurations having larger entropy than black holes is still debated [10].

In close correspondence with entropy maximization by black holes various entropy bounds were formulated for ordinary physical systems. The most appropriate for our purposes is the holographic bound and its derivation via the Susskind process [8,11]. Consider a spherical system of entropy $S$ and surface area $A$ that includes gravity. Let us allow the system to collapse into a black hole. During the collapse the initial entropy $S$ increases to $S_{\mathrm{BH}}$ because of the second law of thermodynamics whereas the confining area $A$ of the system decreases to the black hole event horizon area $A_{\mathrm{BH}}$. That is, $S<S_{\mathrm{BH}}=A_{\mathrm{BH}} / 4<A / 4$ and therefore the entropy of any ordinary system is ultimately bounded by its surface area, $S<A / 4$.

The logic of the Susskind process can be applied to probability distributions with the role of black holes replaced by limit laws. Consider a random variable $x$ with probability density $f(x)$ of finite width but the variance should not necessarily exist. We are interested in the upper bound on the Shannon entropy $S(x)=-\int f(x) \ln f(x) d x$. Making an infinite 
convolution of $f(x)$ with itself while the width being kept fixed a Gaussian or Lévy limit law emerges. Its Shannon entropy is certainly larger than that of $f(x)$ since entropy can not decrease under convolution. A unique property of limit laws is stability: up to a location and scale change their shape remains unmodified under convolution. Since entropy is shift invariant, it is the scale parameter, i.e., the width what represents the Shannon entropy of limit distributions. Therefore $S(x)$ of an arbitrarily chosen probability density is bounded by its width.

Due to the property $S(\lambda x)=S(x)+\log \lambda$ of Shannon entropy it is actually the logarithmic width what measures the entropy of stable laws. The $N$-fold convolution of a $\lambda=1$ stable distribution changes its scale parameter to $\lambda=N^{1 / \alpha}$ where $\alpha$ is the index of stability, $0<\alpha \leq 2$. The $\alpha=2$ case is the Gaussian, otherwise we have a Lévy law with inverse power law tail $x^{-1-\alpha}[6]$. While the width of limit distributions measures the number of stable degrees of freedom being aggregated, the surface area of black holes counts the number of quantum mechanical degrees of freedom on the event horizon $[8,11]$.

The idea that the black hole event horizon might be quantized was suggested by Bekenstein already in the first years of research of BH thermodynamics [12]. In his intuitive picture the horizon surface is tiled with plaquettes of area of the squared Planck length. Although the details of the recent approaches to surface quantization are different, each consistently specifies a quantum of area on the event horizon and hence a quantum of entropy being proportional to it.

A somewhat analogous property characterizes the discrete limit distributions. Aggregating discrete random variables of finite mean the emerging limit law is the Poissonian. The $N$-fold convolution of a Poisson distribution of unit mean reproduces it with mean $N$. Thus the mean of the Poisson law measures the number of aggregated degrees of freedom. Importantly, this holds for non-Poissonian discrete limit laws as well. They can be decomposed into a Poisson distribution of identical clusters. Under convolution only the Poissonian component gets modified (its mean increases) the probability distribution inside the clusters and hence its Shannon entropy remains unchanged. This behavior is the discrete analogue of stability [13]. Therefore the entropy of a non-Poissonian limit law can be thought as quantized: its increase under convolution is due to an increasing number of independent and identical clusters of fixed Shannon entropy. A possible source of discrete stability is the contamination of Lévy fluctuations by Poisson noise. 
The distribution $p(k)$ inside the clusters has a power law tail $k^{-1-\alpha}$. The index $\alpha$ is now restricted to $0<\alpha<1$ and it yields the probability $p(k=1)$. In the limit $\alpha \rightarrow 1$ the pure Poissonian is recovered as the limit distribution. The Shannon entropy of $p(k)$ is fixed by the index $\alpha$ being inversely proportional to it. The role of $\alpha$ bears some resemblance with the Immirzi parameter determining the size of area quanta in the loop quantum gravity approach to black hole entropy [14].

We have seen that the stability property of limit distributions plays a central role in establishing the correspondence with black holes. For continuous limit laws stability expresses a scale symmetry of the same kind what is encountered in renormalization group methods applied to phase transitions [15]. Aggregating random variables is the equivalent of forming block spins whereas scale invariance of the aggregate represents fixed point behavior near the critical point. Therefore the stability of limit distributions is intimately related to the observed similarities between BHs and phase transitions [16,17]. Note also that the Lévy limit laws (continuous or discrete) have power law tails, in accordance with self-similar fluctuations of the event horizon's shape which can be responsible of BH entropy [18]. It is worth considering that two key concepts of the central limit theorem, the $N \rightarrow \infty$ limit of aggregated random variables and the stability of the aggregate's distribution are patterns parallel to the $N \rightarrow \infty$ limit of $S U(N)$ gauge theories and the holographic nature of $\mathrm{BH}$ entropy. The latter are two fundamental ideas behind the AdS/CFT correspondence. Thus one is led to speculate that a probabilistic view of the holographic principle may be also formulated on the basis of the CLT.

Recently it was suggested that a near-horizon conformal symmetry governs black hole thermodynamics, independently of the details of quantum gravity [4,19]. This picture reveals an emergent character of the thermal properties of BHs. The correspondence between limit laws and black holes may ultimately be rooted in an emergent nature of gravity $[17,20]$ with the central limit theorem playing a defining role in it. A less ambitious but more definite view of our result is that, similarly to Hawking radiation, black hole entropy also has analogues in physics outside general relativity, especially in fluctuation phenomena which give birth of stable probability distributions. In any case, it will be nice to see if a new and fruitful interaction develops between probability theory and gravity research. 
$\dagger$ E-mail: hegyi@rmki.kfki.hu

[1] J.D. Bekenstein, Phys. Rev. D 7, 2333 (1973)

[2] S.W. Hawking, Commun. Math. Phys. 43, 199 (1975)

[3] R.M. Wald, Living Rev. Rel. 4, 6 (2001)

[4] S. Carlip, Lect. Notes Phys. 769, 89 (2009)

[5] A.I. Khinchin, Mathematical Foundations of Statistical Mechanics, Dover, New York (1949)

[6] B.V. Gnedenko and A.N. Kolmogorov, Limit Distributions of Sums of Independent Random Variables, Addison-Wesley, Cambridge (1954)

[7] C.E. Shannon, Bell Syst. Tech. J. 27, 349 (1948)

[8] G. 't Hooft, in Salamfestschrift, ed. by A. Ali, J. Ellis and S. Randjbar-Daemi, World-Scientific, Singapore (1994) and arXiv:gr-qc/9310026

[9] R.V. Korkin and I.B. Khriplovich, JETP 95, 1 (2002)

[10] D. Marolf and R.D. Sorkin, Phys. Rev. D 69, 024014 (2004)

J.D. Bekenstein, Phys. Rev. D 70, 121502 (2004)

S.D.H. Hsu and D. Reeb, Phys. Lett. B 658, 244 (2008)

R.D. Sorkin, R.M. Wald and Z.J. Zhang, Gen. Rel. Grav. 13, 1127 (1981)

[11] L. Susskind, J. Math. Phys. 36, 6377 (1995)

[12] J.D. Bekenstein, Lett. Nuovo Cim. 11, 467 (1974)

[13] F.W. Steutel and K. Van Harn, Ann. Probab. 7, 893 (1979)

[14] A. Ashtekar, J. Baez, A. Corichi and K. Krasnov, Phys. Rev. Lett. 80, 904 (1998)

[15] G. Jona-Lasinio, Nuovo Cim. B 26, 99 (1975)

[16] L. Susskind, L. Thorlacius and J. Uglum, Phys. Rev. D 48, 3743 (1993)

[17] R.B. Laughlin, Int. J. Mod. Phys. A 18, 831 (2003)

[18] R.D. Sorkin, in Proc. First Australasian Conference on General Relativity and Gravitation, ed. by D. Wiltshire, University of Adelaide Press, Adelaide (1996) and arXiv:gr-qc/9701056

[19] S. Carlip, Gen. Rel. Grav. 39, 1519 (2007)

[20] O. Dreyer, PoSQG-Ph:016,2007 and arXiv:0710.4350 